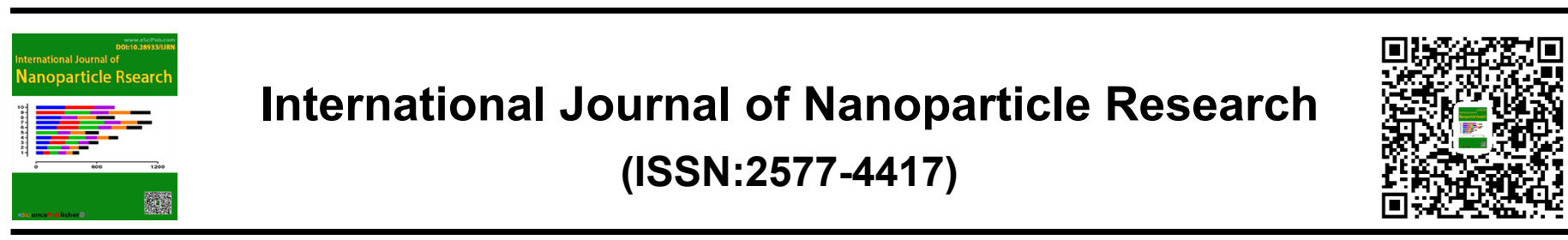

\title{
Fluodot Nanoparticle - A Promising Novel Delivery System For Veterinary Vaccine
}

\section{Aseno Sakhrie ${ }^{1}$, Jingwen Ding ${ }^{2}$, Ankarao Kalluri ${ }^{2}$, Zeinab Helal' ${ }^{1}$, Challa V. Kumar ${ }^{2}$ and} Mazhar I. Khan ${ }^{1 *}$

${ }^{1}$ Department of Pathobiology and Veterinary Science, Institute of Materials Science, University of Connecticut, Storrs CT 06269 USA; '2Department of Chemistry, Department of Molecular and Cell Biology, University of Connecticut, Storrs CT 06269 USA

\section{ABSTRACT}

Most of the killed, inactivated, or live-attenuated pathogen vaccines are now replaced by modern vaccines containing isolated, highly purified antigenic protein subunits which are ${ }^{*}$ Correspondence to Author: safer than live viruses or deactivated viruses. Another strategy Mazhar I. Khan

is the development of nanoparticles which can mimic the Department of Pathobiology, Instirepetitiveness, geometry, size and shape of the host-pathogen surface and provide improved stability and long lasting tute of Materials Science, University of Connecticut, email: mazhar. immunogenicity, as well as serve as vehicles to deliver multiple copies of the antigens to the target cells. Several interesting advances have been made recently in the area of protein nanotechnology and here, we provide a concise review of one such novel nanoparticle called FluoDot which may be effectively used as a delivery system in some veterinary medicine applications.

khan@uconn.edu, Phone: 860486 0228

Keywords: FluoDot; Nanoparticle; Protein: Vaccine

How to cite this article:

Aseno Sakhrie, Jingwen Ding, Ankarao Kalluri, Zeinab Helal, Challa V. Kumar and Mazhar I. Khan. Fluodot Nanoparticle - A Promising Novel Delivery System For Veterinary Vaccine. International Journal of Nanoparticle Research, 2020; 3:14. 


\section{Introduction}

Technological advances especially in the field of nanotechnology have progressed exponentially over the past two decades and have no doubt influenced the field of biology and medical sciences. Nanoparticles are used as a drug carrier system in biomedical science to improve the cellular uptake as well as body distribution. Nanoparticles can be prepared from various materials such as polymers, lipids, polysaccharides and proteins. Protein nanoparticles are non-toxic, biodegradable, easily metabolized, and possess a good biocompatibility, greater stability, and relatively easy to prepare and easy size manipulation ${ }^{[1-5]}$. In biomedical sciences, protein nanoparticle is used in various targeted therapies for cancer ${ }^{[6,7]}$, tumor, cell imaging ${ }^{[8,9]}$ and also as vaccine delivery system against infectious pathogens ${ }^{[19-27]}$. The advantage of using protein nanoparticle-based vaccine is that it prevents the premature degradation, provides good adjuvant properties, improves stability and also aids in targeted delivery of an antigen to the antigen presenting cells (APCs). Single protein nanoparticles are nanoparticles with only one single protein molecule enveloped with a thin shell of polymer or other molecules which are generally highly porous and $3-5 \mathrm{~nm}$ thick ${ }^{[10]}$. This polymer shell protects the protein not only from aggregation but also helps retain its native-like structure ${ }^{11}$. Here we talk about FluoDot nanopar-

ticle, a single protein nanoparticle composed of a central protein core surrounded by a covalently attached lipid layer which helps retain the structure and function of the protein embedded in the core, providing thermal stability and long shelf life. It can be labeled with particular fluorescent dyes for imaging and other applications. They are water-soluble, non-toxic, biocompatible, biodegradable and can be potentially used in LED coatings as biophosphors, in cell imaging or solar cell applications, cancer drug delivery and other biological applications.

\section{Principles of Floudot synthesis}

Floudot is composed of a central protein core of bovine serum albumin (BSA) surrounded by a layer of organic diacid. One of the carboxyl groups of diacid covalently linked to one the many amine groups of BSA by standard carbodiimide coupling chemistry. BSA is a waste product of meat processing, and hence inexpensive and readily available. It is a natural drug delivery vehicle that has the ability to bind a number of different small molecules, dyes, peptides, hormones, and drugs ${ }^{[28]}$. The diacid layer surrounding the protein in the FluoDot mimics the structure of liposome, with one carboxyl group attached to the protein and another exposed to water.
(A)

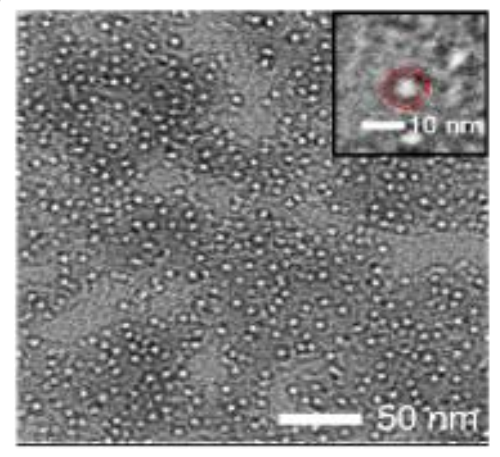

(B)

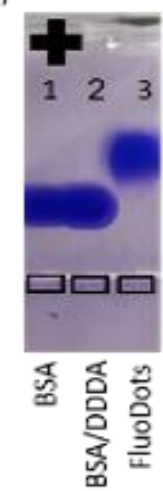

(C)

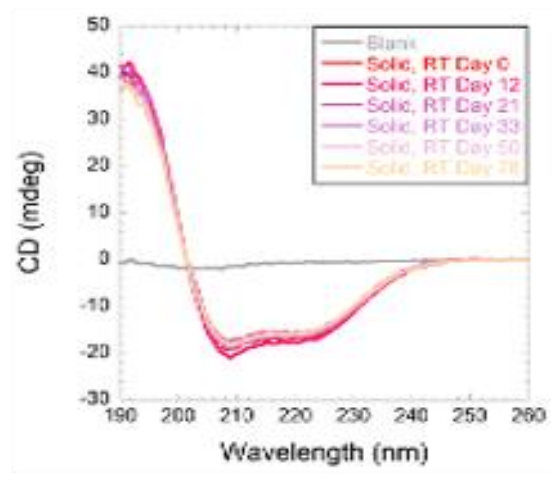

Figure 1. (A) The scanning electron micrograph of the bovine serum albumin-dodecanoic acid FluoDots of $10 \pm 1 \mathrm{~nm}$ diameter (inset). (B) Agarose gel showing the clean conversion of bovine serum albumin to the corresponding FluoDots. (C) The circular dichroism spectra of FluoDots showing near-complete retention of the protein secondary structure and high thermal stability at room temperature (RT) even after $\mathbf{7 0}$ days. These require no coldchain for storage. 
FluoDots are synthesized by activating the diacid with 1-ethyl-3-(3-dimethylaminopropyl) carbodiimide (EDC) and then added to a solution of BSA. The carboxylic acid groups of the diacid covalently link to one of the many amine groups of BSA, as described above. The diacid that was optimized for use was dodecanedioic acid (DDDA). This resulted in a particle a size of $10 \pm 1$ $\mathrm{nm}$ (TEM, Figure $1 \mathrm{~A})$, which are strongly negatively charged (Figure 1B) due to the $\mathrm{COOH}$ groups of the diacids. FluoDots retained the native-like secondary structure of the unmodified BSA, and they are stable even after storing at room temperature for 70 days (Figure $1 \mathrm{C}$ ). The nanocarrier withstood steam sterilization with no loss of protein secondary structure, which is highly unusual, and this stability could be due to the strongly charged diacid shell around the protein protecting the protein from denaturation or aggregation after denaturation.

One of the advantages of Fluodots is their increased thermal stability and a longer shelf life. They are easy to synthesize. FluoDots retain the structure and function of the protein embedded in the core. They are highly soluble in water, biocompatible and biodegradable. Its particle size can be controlled by choosing different lengths of diacids and it can be labelled with appropriate fluorophores. Due to this tunable size and emission properties, a single particle of FluoDot can be labelled with different dyes and used for biosensing and cell imaging or as a vaccine carrier.

\section{Use in veterinary vaccine delivery}

In recent years, there has been a rise in the use of nanoparticles as vaccines against infectious pathogens ${ }^{[20-27]}$. Novel vaccine platforms such as Self-Assembling Protein Nanoparticles (SAPNs) which are composed of protein monomers that are capable of self-assembling into structures that mimic the size and shape of small viruses has been successfully developed as potent vaccine prototypes for Severe Acute Respiratory Syndrome [21], HIV [24], toxoplasmosis [25], malaria ${ }^{[23]}$ influenza ${ }^{[22,27]}$ and infectious bronchitis virus ${ }^{[19]}$. Infectious bronchitis virus (IBV) is a highly prevalent coronavirus of chickens which is the foremost cause of economic loss to the US poultry industry affecting the performance of both meat-type and egg-laying birds. There are several limitations for using live attenuated virus ${ }^{[12]}$ and inactivated viruses ${ }^{[13,14]}$ for IBV vaccine production. DNA subunit and synthetic peptides alone have been used as vaccines but they have limited protection and required boosters [15-18]. FluoDot nanoparticles for delivery of antigenic peptide of IBV vaccine have been explored in our laboratories. FluoDot conjugated with a short peptide sequence which is part of the $S$ protein of IBV showed the formation of antibodies corresponding to IBV-FluoDots, and protected chickens against challenge with Massachusetts 41 (M41) field type IBV. Five groups of chickens were immunized at four weeks of age with the vaccine IBV-FluoDots, a negative buffer control, a Fluo- Dot control, a IBV peptide control and an inactivated vaccine as positive control. The immunized chickens were challenged with $2 \times 10^{4.74}$ EID 50 IBV M41 strain. Antibody was detected in chickens immunized with IBV-FluoDot and in ex vivo proliferation tests, peripheral mononuclear cells (PBMCs) derived from IBV-FluoDot immunized chickens had a higher stimulation index than that of PBMCs from chickens receiving inactivated vaccine control. Real time RT-PCR results indicated that after injection of IBVFluoDots challenged with IBV viral infection in chickens, virus shedding was reduced in vaccinated chickens with IBV-FlouDots. Further improvements to the potential vaccine can be made by attaching multiple epitopes to the FluoDot surface or via other refinements such as the use of full length $S 1 / \mathrm{S} 2$ protein or multiple virus particles to be conjugated to the nanoparticle. Such conjugates with multiple copies of multiple epitopes would serve as multi-serotype vaccines with a high potential for success.

\section{Conclusions}

FlouDots are novel single protein fluorescent nanoparticles, which have the potential to be used as a delivery system for vaccines in chickens but further studies are required.

\section{Acknowledgement}

Authors acknowledge the financial support from UCONN START grant from the Office of the Vice 
Provost for Research, USDA-Hatch grant, Storrs

Experiment Station. We are also thankful to the Ministry of Tribal affairs, Government of India for providing Aseno Sakhire scholarship to earn her $\mathrm{PhD}$ degree.

\section{Conflicts of interest}

\section{None}

\section{References}

[1] Crivelli B, Perteghella S, Bari E, Sorrenti M, Tripodo G, Chlapanidas T, Torre M L. (2018) Silk Nanoparticles: From Inert Supports to Bioactive Natural Carriers for Drug Delivery. Soft Matter, 14 (4), 546-557. DOI:10.1039/C7SM01631J.

[2] Verma D, Gulati N, Kaul S, Mukherjee S, Nagaich U. (2018). Protein Based Nanostructures for Drug Delivery. J. Pharm. 2018, 9285854. DOI:10.1155/2018/9285854.

[3] Zhang Y, Sun T, Jiang C. (2018). Biomacromolecules as Carriers in Drug Delivery and Tissue Engineering. Acta Pharm. Sin. B, 8 (1), 34-50. DOI:10.1016/j.apsb.2017.11.005.

[4] Lee E J, Lee N K, Kim I S. Bioengineered Protein-Based Nanocage for Drug Delivery.Adv. Drug Deliv. Rev.2016, 106 (Pt A), 157-171. DOI:10.1016/j.addr.2016.03.002.

[5] Salatin S, Jelvehgari M, Maleki-Dizaj S, Adibkia K. (2015) A Sight on Protein-Based Nanoparticles as Drug/Gene Delivery Systems. Ther. Deliv. 6 (8), 1017-1029. DOI:10.4155/tde.15.28.

[6] Ferrer-Miralles N, Rodriguez-Carmona E, Corchero JL, et al. (2015) Engineering protein selfassembling in protein-based nanomedicines for drug delivery and gene therapy. Crit Rev Biotechnol. 35(2):209-221.

[7] Jeong Y, Jo YK, Kim BJ, Yang B, Joo KI, Cha HJ. (2018)Sprayable Adhesive Nanotherapeutics: Mussel-Protein-Based Nanoparticles for Highly Efficient Locoregional Cancer Therapy. ACS Nano. 2018;12(9):8909-8919. doi:10.1021/ acsnano.8b04533

[8] Lin X, Xie J, Zhu L, et al. (2011) Hybrid ferritin nanoparticles as activatable probes for tumor imaging. Angew Chem Int Ed Engl. 50(7):15691572. doi:10.1002/anie.201006757

[9] Stromer BS, Roy S, Limbacher MR, et al. (2018). Multicolored Protein Nanoparticles: Synthesis, Characterization, and Cell Uptake. Bioconjug Chem.29(8):2576-2585. doi:10.1021/acs.bioconjchem. 8b00282

[10] Kim J, Grate JW. (2003). Single-enzyme nanoparticles armored by a nanometer-scale organic/inorganic network. Nano Letters. 3(9), pp. 1219-1222.

[11] Sharma RK, Das S, Maitra A. (2005). Enzymes in the cavity of hollow silica nanoparticles.
Author information. Journal of Colloid and Interface Science. 284(1):358-361. DOI: 10.1016/j.jcis.20

04.10.006 PMID: 15752825

[12] Tarpey I, Orbell S, Britton P, Casais R, Hodgson T, Lin F, et al. (2006) Safety and efficacy of an infectious bronchitis virus used for chicken embryo vaccination. Vaccine. 24: 6830-6838. Pmid: 16860445

[13] Collisson EW, Pei J, Dzielawa J, Seo SH. (2000). Cytotoxic T lymphocytes are critical in the control of infectious bronchitis virus in poultry. Deve-

lopmental \& Comparative Immunology. 24:187200.

[14] Ladman B, Pope C, Ziegler A, Swieczkowski T, Callahan J, Davison S, et al. (2002). Protection of chickens after live and inactivated virus vaccination against challenge with nephropathogenic infectious bronchitis virus PA/Wolgemuth/98. Avian Dis. 46: 938-944. pmid:124950 55

[15] Babapoor S, Almeida D, Fabis JJ, Helal Z, Wang X, Girshick T and Khan MI. (2009). Protective Effect of In ovoVaccination with IBV-Spike Recombinant DNA and Chicken Interferon as an Adjuvant. Int. J. Poult Sci. 8 (11): 1034-1041.

[16] Jackwood MW, Hilt DA. (1995). Production and immunogenicity of multiple antigenic peptide (MAP) constructs derived from S1 glycoprotein of infectious bronchitis virus (IBV). Adv Exp. Med. Biol. 380 213-219.

[17] Wang X, Schnitzlein WM, Tripathy DN, Girshick T and Khan MI, (2001). Construction and immunogenicity studies of recombinant fowl poxvirus containing the S1 gene of Massachusetts 41 strain of infectious bronchitis virus. Avian Dis. 46: 831-838.

[18] Wang L, Parr RL, King DJ, Collisson EW. (1995) A highly conserved epitope on the spike protein of infectious bronchitis virus. Archives of Virology. 140:12, pp 2201-2213.

[19] Li J, Helal ZH, Karch CP, Mishra N, Girshick T, Garmendia A, et al. (2018). A self-adjuvanted nanoparticle based vaccine against infectious bronchitis virus. PLoS ONE. 13(9): e0203771. https://doi.org/10.1371/journal.pone.0203771

[20] Kaba SA, Brando C, Guo Q, Mittelholzer C, Raman S, Tropel D, et al. (2009). A nonadjuvanted polypeptide nanoparticle vaccine confers longlasting protection against rodent malaria. $\mathrm{J} \mathrm{Im}$ munol. ;183: 7268-7277. Pmid:19915055

[21] Pimentel TA, Yan Z, Jeffers SA, Holmes KV, Hodges RS, Burkhard P. (2009). Peptide nanoparticles as novel immunogens: design and analysis of a prototypic severe acute respiratory 
syndrome vaccine. Chemical biology \& drug design. 73: 53-61.

[22] Babapoor S, Neef T, Mittelholzer C, Girshick T, Garmendia A, Shang H, et al. (2011). A Novel Vaccine Using Nanoparticle Platform to Present Immunogenic M2e against Avian Influenza Infection. Influenza Res Treat. 2011: 126794. pmid:23074652

[23] Kaba SA, McCoy ME, Doll TA, Brando C, Guo $Q$, Dasgupta D, et al. Protective antibody and CD8 T-cell responses to the Plasmodium falciparum circumsporozoite protein induced by a nanoparticle vaccine. PLoS One. 2012;7:e4830 4. pmid:23144750

[24] Wahome N, Pfeiffer T, Ambiel I, Yang Y, Keppler OT, Bosch V, et al. (2012). Conformation-specific Display of 4E10 and 2F5 Epitopes on Selfassembling Protein Nanoparticles as a Potential HIV Vaccine. Chemical biology \& drug design. 80: 349-357.

[25] El Bissati K, Zhou Y, Dasgupta D, Cobb D, Dubey JP, Burkhard P, et al. (2014). Effectiveness of a novel immunogenic nanoparticle platform for Toxoplasma peptide vaccine in HLA transgenic mice. Vaccine. 32: 3243-3248. pmid:24736000

[26] Thrane S, Janitzek CM, Agerbæk MØ, Ditlev SB, Resende M, Nielsen MA, et al. (2015). A novel virus-like particle based vaccine platform displaying the placental malaria antigen VAR2CSA. PLoS One. 10: e0143071. pmid:26599509

[27] Karch CP, Li J, Kulangara C, Paulillo SM, Raman SK, Emadi S, et al. (2017). Vaccination with self-adjuvanted protein nanoparticles provides protection against lethal influenza challenge. Nanomedicine: Nanotechnology, Biology and Medicine. 13: 241-251.

[28] Lohcharoenkal W, Wang L, Chen Y C and Rojanasakul Y. (2014). Protein nanoparticles as drug delivery carriers for cancer therapy Biomed. Res. Int. 180549. 